\title{
BIPOLAR MAGNETIC SPOTS FROM DYNAMOS IN STRATIFIED SPHERICAL SHELL TURBULENCE
}

\author{
Sarah Jabbari ${ }^{1,2}$, Axel Brandenburg ${ }^{1,2}$, NAthan KleEorin ${ }^{1,3}$, Dhrubaditya Mitra ${ }^{1}$, And IgOr RogachevskiI ${ }^{1,3}$ \\ ${ }^{1}$ Nordita, KTH Royal Institute of Technology and Stockholm University, Roslagstullsbacken 23, SE-10691 Stockholm, Sweden; sarahjab@kth.se \\ ${ }^{2}$ Department of Astronomy, AlbaNova University Center, Stockholm University, SE-10691 Stockholm, Sweden \\ ${ }^{3}$ Department of Mechanical Engineering, Ben-Gurion University of the Negev, POB 653, Beer-Sheva 84105, Israel \\ Received 2014 November 18; accepted 2015 April 4; published 2015 May 29
}

\begin{abstract}
Recent work by Mitra et al. (2014) has shown that in strongly stratified forced two-layer turbulence with helicity and corresponding large-scale dynamo action in the lower layer, and nonhelical turbulence in the upper, a magnetic field occurs in the upper layer in the form of sharply bounded bipolar magnetic spots. Here we extend this model to spherical wedge geometry covering the northern hemisphere up to $75^{\circ}$ latitude and an azimuthal extent of $180^{\circ}$. The kinetic helicity and therefore also the large-scale magnetic field are strongest at low latitudes. For moderately strong stratification, several bipolar spots form that eventually fill the full longitudinal extent. At early times, the polarity of spots reflects the orientation of the underlying azimuthal field, as expected from Parker's $\Omega$-shaped flux loops. At late times their tilt changes such that there is a radial field of opposite orientation at different latitudes separated by about $10^{\circ}$. Our model demonstrates the spontaneous formation of spots of sizes much larger than the pressure scale height. Their tendency to produce filling factors close to unity is argued to be reminiscent of highly active stars. We confirm that strong stratification and strong scale separation are essential ingredients behind magnetic spot formation, which appears to be associated with downflows at larger depths.
\end{abstract}

Key words: magnetohydrodynamics (MHD) - Sun: activity - dynamo - turbulence

\section{INTRODUCTION}

Solar activity is characterized by the formation of magnetic spots. Sunspots are relatively small concentrations of magnetic field at the surface, where the radiation is suppressed significantly, making these regions cooler than their surroundings. Similar phenomena are also expected to occur on other stars exhibiting magnetic cycles, although the starspots observed so far all tend to be significantly larger than sunspots and not necessarily spatially correlated with the surface temperature (Kochukhov et al. 2013). There is little doubt that magnetic spots are associated with an underlying dynamo in the outer convection zones of these stars, but it is not clear whether they are caused by deeply rooted magnetic flux tubes at the bottom of the convection zones (Caligari et al. 1995), or whether they are merely shallow magnetic concentrations formed locally where the near-surface magnetic field exceeds a certain threshold. Magnetic field visualizations from convectively driven dynamo simulations have shown serpentineshaped flux tubes that can be expected to intersect the surface (Fan \& Fang 2014; Nelson \& Miesch 2014), but these tubes would expand during their ascent, so some sort of reamplification of these tubes would be needed to explain sunspots.

The foundations of magnetic spot formation have been developed by Parker (1955), who identified magnetic buoyancy as the main agent responsible for bringing magnetic fields to the surface. In his subsequent work, Parker (1979) identified the need for a special mechanism to make these concentrations sufficiently cool and evacuated so as to explain the observed values of the magnetic field in sunspots. He postulated the existence of suitable downflows that would help to evacuate the magnetic flux tube in its upper parts.

The appearance of such downdrafts in the region of spontaneously formed magnetic spots has been observed in the numerical simulations of Brandenburg et al. (2013) using forced turbulence with weak imposed vertical magnetic field.
Downdrafts have also been seen in simulations of buoyantly rising flux tubes some time after they reached the surface (Rempel \& Cheung 2014). A possible mechanism for producing such downflows might well be the negative effective magnetic pressure instability (NEMPI). It is based on the magnetic suppression of the total (hydrodynamic plus magnetic) turbulent pressure. The importance of the difference in turbulent pressure inside and outside magnetic structure was first emphasized by van Ballegooijen (1984). Subsequent mean-field calculations have shown that, if the magnetic Reynolds number is larger than unity, the effective large-scale magnetic pressure (sum of turbulent and non-turbulent contributions) becomes negative and a large-scale instability (namely NEMPI) can be excited (Kleeorin et al. 1989, 1990, 1996; Kleeorin \& Rogachevskii 1994; Rogachevskii \& Kleeorin 2007). This instability redistributes magnetic flux and can cause the formation of magnetic structures. As the work of recent years has shown, for horizontal magnetic fields, negative effective magnetic pressure leads to negative magnetic buoyancy at sub-equipartition field strengths (Brandenburg et al. 2010, 2011). However, for vertical fields the return flow replenishing the downflow occurs predominantly along magnetic field lines and has a much larger impact near the surface, where it can lead to super-equipartition strength flux concentrations; Brandenburg et al. 2013). Corresponding mean-field simulations (MFS; Brandenburg et al. 2014) have displayed great similarity with Parker's original picture (Parker 1979), where he explicitly stated the need for postulating the existence of downdrafts, leaving however the question about their origin open. On the other hand, the downflows have strengths of only about $20 \%$ of the turbulent rms velocity and are therefore not easily recognized among the downflows due to convection. Depending on circumstances, NEMPI can also lead to the formation of bipolar spots (Warnecke et al. 2013, 2015) with super-equipartition field strengths (Mitra et al. 2014). The latter looks remarkably similar to the bipolar regions found by Stein 
$\&$ Nordlund (2012) in realistic simulations of solar convection with an unstructured magnetic field being supplied at the bottom of their domain.

Strong stratification is a prerequisite for NEMPI to occur. In recent direct numerical simulations (DNS) of Mitra et al. (2014), it was demonstrated that stratification plays a crucial role in the formation of magnetic spots that have surprisingly sharp boundaries with opposite polarities on the two sides. In these simulations, the turbulence in the deeper parts was made helical such that a large-scale magnetic field can be generated by the $\alpha$ effect associated with the kinetic helicity of the turbulence. In the upper part of the domain in the DNS of Mitra et al. (2014) the forcing was non-helical, so there is no $\alpha$ effect, but NEMPI can still work. What is surprising in those simulations is the fact that the magnetic fields in these structures exceeds the equipartition value by a factor of three or more. Since the effective magnetic pressure has not been measured in the simulations of Mitra et al. (2014), it is not obvious that the underlying mechanism is actually related to NEMPI, even though downflows with a strength of about $20 \%$ of the turbulent velocity have been detected by Mitra et al. (2014). The physics of the formation of sharp magnetic edges of bipolar structures in the turbulent flow remains however elusive.

The magnetic field from the dynamo simulations of Mitra et al. (2014) had the property of extending over the entire horizontal length of the domain. As a consequence, only one bipolar structure was produced, which occasionally developed horizontal bands extending again over the full length of the horizontally periodic domain. To overcome this artifact of periodic boundary conditions, it is important to consider larger domains with either no or at least with physically motivated boundary conditions. A spherical shell is an obvious choice.

The dynamics of NEMPI from dynamo-generated magnetic fields in spherical shells or wedges has recently been studied by Jabbari et al. (2013) in a MFS, although in their case the dynamo mechanism operated throughout the domain and not just below a certain depth, as in the DNS of Mitra et al. (2014). Here we combine the two-layer setup of Mitra et al. (2014) with the shell geometry used in the MFS of Jabbari et al. (2013). There is also another DNS study by Jabbari et al. (2014) in which they investigated a combined system of dynamo and NEMPI in Cartesian geometry. In that paper, the combination of rotation and stratification leads to an $\alpha^{2}$ dynamo. In the present work, we ignore rotation to understand first a simpler case using instead helically forced turbulence. Investigation of a similar system with rotation and shear will be the subject of a future study.

The purpose of the present paper is to study forced turbulence in a strongly stratified spherical shell. As in Mitra et al. (2014), the turbulence is made helical below a certain radius $r_{\star}$ so as to enable the formation of large-scale magnetic fields by the $\alpha^{2}$ dynamo mechanism. The sign of the helicity is assumed to change across the equator. This leads to the formation of dynamo waves that travel toward the equator with opposite polarity in the southern hemisphere (Mitra et al. 2010). This is a property that has been associated with the choice of perfectly conducting boundary conditions at high latitudes of the wedge. On the other hand, changing the highlatitude boundary condition to a normal field condition (often referred to as a vertical field condition) causes dynamo waves to propagate away from the equator and toward high latitudes, but now with the same polarity in both hemispheres (Brandenburg et al. 2009). In the present paper, we reconsider the former case and apply a suitable boundary condition at the equator to cut the computational costs.

There is another potential artifact of the dynamo waves of Mitra et al. (2010) in that they tend to occur at high latitudes where the kinetic helicity is assumed largest. On the other hand, we have seen in DNS of turbulent convection in spherical shells that kinetic helicity is in fact concentrated to regions outside the inner tangent cylinder of the shell (Käpylä et al. 2012b). This restricts the kinetic helicity essentially to low latitudes below $45^{\circ}$. We model this feature here by assuming a suitable profile for the kinetic helicity of the forcing function in the deeper parts of the shell.

\section{THE MODEL}

In this paper we investigate a system similar to that of Mitra et al. (2014), but in spherical geometry assuming either symmetric (quadrupolar) or anti-symmetric (dipolar) field properties about the equator. Jabbari et al. (2013) used MFS in spherical geometry to show how the large-scale dynamo can interact with NEMPI in such a coupled system. In their MFS, the mean-field Lorentz force was parameterized, which is subject to uncertainties. It is therefore useful to perform DNS and to study how the results depend on domain size, density stratification, geometry, and boundary conditions. As explained above, the main difference here is the fact that the forcing is not uniform in whole domain. As in Mitra et al. (2014), our domain is divided into two parts. We apply helical forcing (which leads to an alpha squared dynamo) in the lower part of the domain and non-helical forcing in the upper part of the domain. The position of the border between these two areas is varied to see how it affects the results. We expect to detect similar intense bipolar region of earlier DNS of Mitra et al. (2014).

We use an isothermal equation of state, so no convection is possible. Therefore, turbulence is driven using volume forcing given by a function $f$ that is $\delta$-correlated in time and monochromatic in space. It consists of random non-polarized waves whose direction and phase change randomly at each time step. We present a more detailed discussion about forcing in Section 2.2.

\subsection{Basic Equations}

In DNS of an isothermally stratified layer we solve the equations for the velocity $\boldsymbol{U}$, the magnetic vector potential $\boldsymbol{A}$, and the density $\rho$,

$$
\begin{gathered}
\rho \frac{D \boldsymbol{U}}{D t}=\boldsymbol{J} \times \boldsymbol{B}-c_{s}^{2} \nabla \rho+\nabla \cdot(2 \nu \rho \mathbf{S})+\rho(\boldsymbol{f}+\boldsymbol{g}), \\
\frac{\partial \boldsymbol{A}}{\partial t}=\boldsymbol{U} \times \boldsymbol{B}+\eta \nabla^{2} \boldsymbol{A}, \\
\frac{\partial \rho}{\partial t}=-\nabla \cdot \rho \boldsymbol{U},
\end{gathered}
$$

where the operator $D / D t=\partial / \partial t+\boldsymbol{U} \cdot \nabla$ is the advective derivative, $\eta$ is the magnetic diffusivity, $\boldsymbol{B}=\boldsymbol{\nabla} \times \boldsymbol{A}$ is the magnetic field, $\boldsymbol{J}=\boldsymbol{\nabla} \times \boldsymbol{B} / \mu_{0}$ is the current density, $\mathrm{S}_{i j}=\frac{1}{2}\left(U_{i, j}+U_{j, i}\right)-\frac{1}{3} \delta_{i j} \nabla \cdot \boldsymbol{U}$ is the traceless rate of strain tensor (the commas denote partial differentiation), $\nu$ is the 
kinematic viscosity, $c_{s}$ is the isothermal sound speed, and $\mu_{0}$ is the vacuum permeability. We adopt spherical coordinates $(r, \theta, \phi)$.

For the $\phi$ direction we use periodic boundary conditions. In the radial direction (the direction of the stratification), we consider perfectly conducting boundary conditions at the bottom and a vertical field boundary condition at the top. At the equator, we adopt a symmetry condition such that the magnetic field is either symmetric (quadrupolar) or antisymmetric (dipolar) with respect to the equator. For the magnetic field we assume perfect conductor boundary conditions on the latitudinal $\left(\theta=\theta_{0}\right)$ and lower radial $\left(r=r_{0}\right)$ boundaries, and radial field boundary conditions on the outer radius $(r=R)$. On the equator, we assume either dipolar or a quadrupolar symmetry. In terms of the magnetic vector potential these conditions translate to

$$
\begin{gathered}
\frac{\partial A_{r}}{\partial r}=A_{\theta}=A_{\phi}=0 \quad\left(r=r_{0}\right), \\
A_{r}=0, \quad \frac{\partial A_{\theta}}{\partial r}=-\frac{A_{\theta}}{r}, \quad \frac{\partial A_{\phi}}{\partial r}=-\frac{A_{\phi}}{r} \quad(r=R), \\
A_{r}=\frac{\partial A_{\theta}}{\partial \theta}=A_{\phi}=0 \quad\left(\theta=\theta_{0}, \pi / 2\right)
\end{gathered}
$$

for quadrupolar symmetry and

$$
\frac{\partial A_{r}}{\partial \theta}=A_{\theta}=\frac{\partial A_{\phi}}{\partial \theta}=0 \quad(\theta=\pi / 2)
$$

for dipolar symmetry.

For the velocity field we use stress-free, non-penetrating boundary conditions in the radial direction. The gravitational acceleration is $g=-\nabla \Phi$, where

$$
\Phi(r)=-G M\left(\frac{1}{r}-\frac{1}{r_{m}}\right) .
$$

Here $G$ is Newton's constant and $M$ is the mass of the sphere (or star). For an isothermal gas, the hydrostatic density stratification obeys $\rho=\rho_{0} \exp \left(-\Phi / c_{s}{ }^{2}\right)$, where $\rho=\rho_{0}$ is the density in the middle of the shell at $r=r_{m}=\left(r_{0}+R\right) / 2$. The radial component of the gravitational acceleration is then $g=-G M / r^{2}$. The quantity $G M$ determines the density contrast $\Gamma_{\rho}=\rho_{\text {bot }} / \rho_{\text {top }}$ between bottom and top of the domain. Initially, we have $\Gamma_{\rho}=\exp \left(R / r_{0}-1\right)^{G M / R c_{s}^{2}}$. The density scale height is given by $H_{\rho}=c_{s}^{2} / G M$. The thickness of the shell is $\Delta r=R-r_{0}$, and it is used to define a reference wavenumber $k_{1}=2 \pi / \Delta r$.

\subsection{The Forcing Function}

The forcing function $f$ is similar to that of Mitra et al. (2014),

$$
\boldsymbol{f}(\boldsymbol{x}, t)=\operatorname{Re}[N \tilde{\boldsymbol{f}}(\boldsymbol{k}, t) \exp (i \boldsymbol{k} \cdot \boldsymbol{x}+i \varphi)],
$$

where $\boldsymbol{x}$ is the position vector, $-\pi<\varphi \leqslant \pi$ is a randomly selected phase, and $\boldsymbol{k}$ is the wavevector which is chosen from a set of wavevectors in a certain range around a given forcing wavenumber, $k_{f}$. The Fourier amplitudes, $\tilde{\boldsymbol{f}}(\boldsymbol{k})$, are defined as

$$
\tilde{\boldsymbol{f}}(\boldsymbol{k})=\mathbf{R} \cdot \tilde{\boldsymbol{f}}(\boldsymbol{k})^{(\text {nohel })} \quad \text { with } \quad \mathrm{R}_{i j}=\frac{\delta_{i j}-i \sigma \epsilon_{i j k} \hat{k}}{\sqrt{1+\sigma^{2}}},
$$

where $\sigma$ characterizes the fractional helicity of $\boldsymbol{f}$, and

$$
\tilde{\boldsymbol{f}}(\boldsymbol{k})^{(\text {nohel })}=(\boldsymbol{k} \times \hat{e}) / \sqrt{\boldsymbol{k}^{2}-(\boldsymbol{k} \cdot \hat{e})^{2}}
$$

is a non-helical forcing function, and $\hat{e}$ is an arbitrary unit vector not aligned with $\boldsymbol{k}$ and $\hat{\boldsymbol{k}}$ is the unit vector along $\boldsymbol{k}$; note that $|\tilde{f}|^{2}=1$. The degree of helicity is modulated in space via the function

$$
\sigma(r, \theta)=\frac{\sigma_{\max }}{2}\left[1-\operatorname{erf}\left(\frac{r-r_{*}}{w_{f}}\right)\right] \cos \theta \sin ^{n} \theta
$$

where erf is the error function, $r_{*}$ is the radius above which the helicity vanishes, $w_{f}$ is the width of the transition layer, and the exponent $n$ determines the latitudinal helicity profile. We choose $w_{f}=0.01$ for all the simulations. The amplitude of the forcing is, however, independent of $r$ and therefore also the rms velocity is essentially independent of $r$. For more details of this type of forcing see Mitra et al. (2014).

We note that the degree of helicity of the forcing function is here assumed to be independent of the degree of stratification. In reality, of cause, helicity is actually a consequence of stratification together with rotation (Krause \& Rädler 1980). We return to this question in the conclusions, where we discuss possible artifacts resulting from this assumption.

\subsection{Parameters of the Simulations}

During the exponential growth phase of the dynamo, the growth rate is calculated as $\lambda=d \ln B_{\text {rms }} / d t$. The non-dimensional growth rate is given as $\tilde{\lambda}=\lambda / u_{\mathrm{rms}} k_{f}$. However, the time of the simulation is normally specified in terms of the turbulentdiffusive time $\tau_{\mathrm{td}}=\left(\eta_{\mathrm{t} 0} k_{1}^{2}\right)^{-1}$, where $\eta_{\mathrm{t} 0}=u_{\mathrm{rms}} / 3 k_{f}$ is the estimated turbulent diffusivity. In most of the calculations, we use a scale separation ratio $k_{f} / k_{1}$ of 30 and a fluid Reynolds number $\operatorname{Re} \equiv u_{\mathrm{rms}} / \nu k_{f}$ of 20 . Our magnetic Prandtl number $\operatorname{Pr}_{M}=\nu / \eta$ is 1 , so the magnetic Reynolds number is then $\operatorname{Re}_{M}=\operatorname{Pr}_{M} \operatorname{Re}=20$. These values are chosen to have both $k_{f}$ and Re large enough for NEMPI to develop at an affordable numerical resolution. The magnetic field is expressed in units of local equipartition magnetic field, $B_{\text {eq }}(r)=\sqrt{\mu_{0} \bar{\rho}(r)} u_{\mathrm{rms}}$, where $\bar{\rho}(r)$ is the density averaged over time and spherical shells. We also define $B_{\text {eq } 0}=\sqrt{\mu_{0} \rho_{0}} u_{\mathrm{rms}}$. In the following, we use non-dimensional units by setting $c_{s}=\mu_{0}=\rho_{0}=1$.

We perform simulations with values of $G M / R c_{s}^{2}$ between 1 and 17. With $\exp \left(R / r_{0}-1\right) \approx 1.54$, this implies that $\Gamma_{\rho} \approx 1.54^{G M / R c_{s}^{2}}$ between 1.5 and 1460 for the initial values. In the following, however, we quote the values from the relaxed run. We perform simulations with different values of $\Gamma_{\rho}$, which enables us to study the effect of stratification on the formation of magnetic structures. The corresponding stratification parameter of Jabbari et al. (2014), $\mathrm{Gr}=\left(k_{f} H_{\rho}\right)^{-1}$, varies then between 0.002 (for $G M / R c_{s}^{2}=1$ ) and 0.03 (for $\left.G M / R c_{s}^{2}=17\right)$. Even the latter value is still rather small compared with the value of 0.16 expected from solar mixing 
Table 1

Summary of the Runs. The Reference Run is Shown in Bold

\begin{tabular}{lrlcccccc}
\hline \hline Run & $\Gamma_{\rho}$ & $r_{\star}$ & $\phi$ ext. & $\mathrm{Re}_{M}$ & $n$ & b.c. & $\sigma_{\max }$ & $\tilde{\lambda}$ \\
\hline Q1 & 2 & 0.8 & $\pi$ & 20 & 6 & $\mathrm{Q}$ & 1 & 0.079 \\
Q1b & 30 & 0.8 & $\pi$ & 20 & 6 & $\mathrm{Q}$ & 1 & 0.085 \\
Q1c & 70 & 0.8 & $\pi$ & 20 & 6 & $\mathrm{Q}$ & 1 & 0.083 \\
Q2 & $\mathbf{4 5 0}$ & $\mathbf{0 . 8}$ & $\pi$ & $\mathbf{2 0}$ & $\mathbf{6}$ & $\mathbf{Q}$ & $\mathbf{1}$ & $\mathbf{0 . 0 8 4}$ \\
Q3 & 1400 & 0.8 & $\pi$ & 20 & 6 & $\mathrm{Q}$ & 1 & 0.074 \\
Q4 & 450 & 0.75 & $\pi$ & 20 & 6 & $\mathrm{Q}$ & 1 & 0.074 \\
D1 & 2 & 0.8 & $\pi$ & 20 & 6 & $\mathrm{D}$ & 1 & 0.087 \\
D2 & $\mathbf{4 5 0}$ & $\mathbf{0 . 8}$ & $\pi$ & $\mathbf{2 0}$ & $\mathbf{6}$ & $\mathbf{D}$ & $\mathbf{1}$ & $\mathbf{0 . 0 8 2}$ \\
D3 & 1400 & 0.8 & $\pi$ & 20 & 6 & $\mathrm{D}$ & 1 & 0.077 \\
D4 & 450 & 0.8 & $\pi$ & 20 & 6 & $\mathrm{D}$ & 0.2 & 0.11 \\
D5 & 450 & 0.8 & $\pi$ & 20 & 6 & $\mathrm{D}$ & 0.5 & 0.11 \\
R1 & 450 & 0.8 & $\pi / 4$ & 20 & 6 & $\mathrm{D}$ & 1 & 0.0083 \\
R2 & 450 & 0.8 & $\pi / 4$ & 40 & 6 & $\mathrm{D}$ & 1 & 0.075 \\
H1 & 2 & 0.8 & $\pi$ & 20 & 0 & $\mathrm{Q}$ & 1 & 0.087 \\
H2 & 450 & 0.8 & $\pi$ & 20 & 0 & $\mathrm{Q}$ & 1 & 0.083 \\
H3 & 1400 & 0.8 & $\pi$ & 20 & 0 & $\mathrm{Q}$ & 1 & 0.076 \\
H4 & 450 & 0.75 & $\pi$ & 20 & 0 & $\mathrm{Q}$ & 1 & 0.081 \\
\hline
\end{tabular}

Note. The column "b.c." indicates whether the equatorial boundary condition is dipolar (D) or quadrupolar (Q).

length theory. Increasing the value of Gr leads to a slight decrease of the growth rate of NEMPI compared with the theoretically expected value; see Jabbari et al. (2014) for details.

For most of the simulations, we choose $n=6$ in Equation (12), i.e., the helicity is maximum at lower latitudes. This is also the case for our two reference runs, which have $\Gamma_{\rho}=450$ and either quadrupolar or dipolar parity. However, for comparison we also present cases where $n=0$.

We use the Pencil CODE $^{4}$ to perform DNS. This code uses sixth-order explicit finite differences in space and a third-order accurate time-stepping method. We use $r_{0}=0.7 R$ and $\theta_{0}=15^{\circ}$. For runs with a $\phi$ extend of $\pi$, we use a numerical resolution of $256 \times 1152 \times 1152$ mesh points in the $r, \theta$, and $\phi$ directions, and $256 \times 1152 \times 288$ for all other runs. Table 1 shows all runs with their parameters.

\section{RESULTS}

\subsection{Nature of the Dynamo}

MFS of $\alpha^{2}$ dynamos in spherical wedges have shown that the magnetic field is strongest near the high-latitude boundaries (Jabbari et al. 2013). However, in rapidly rotating stratified spherical shell convection, the kinetic helicity is typically found to be maximum close to the equator, e.g., at $\pm 15^{\circ}$ latitude (Käpylä et al. 2012b). For this reason, we focus in the present paper on the case $n=6$, which yields a maximum of the magnetic field at about $22^{\circ}$.

In Figure 1 we show butterfly diagrams of $B_{r}(\theta, t) / B_{\text {eq }}$ at $r / R=0.75,0.8$, and 0.95 and $\phi=0$. We observe equatorward migration of the magnetic field at low latitudes (below 20 $0^{\circ}$ and poleward migration at higher ones. Note, however, that the latitudinal variation of the magnetic field is much more complex than the field variations of similar mean-field calculations (Jabbari et al. 2013) and even DNS with forced unstratified turbulence (Warnecke et al. 2011). A possible reason for this can be the larger aspect ratio of the dynamo-

\footnotetext{
http://pencil-code.googlecode.com
}
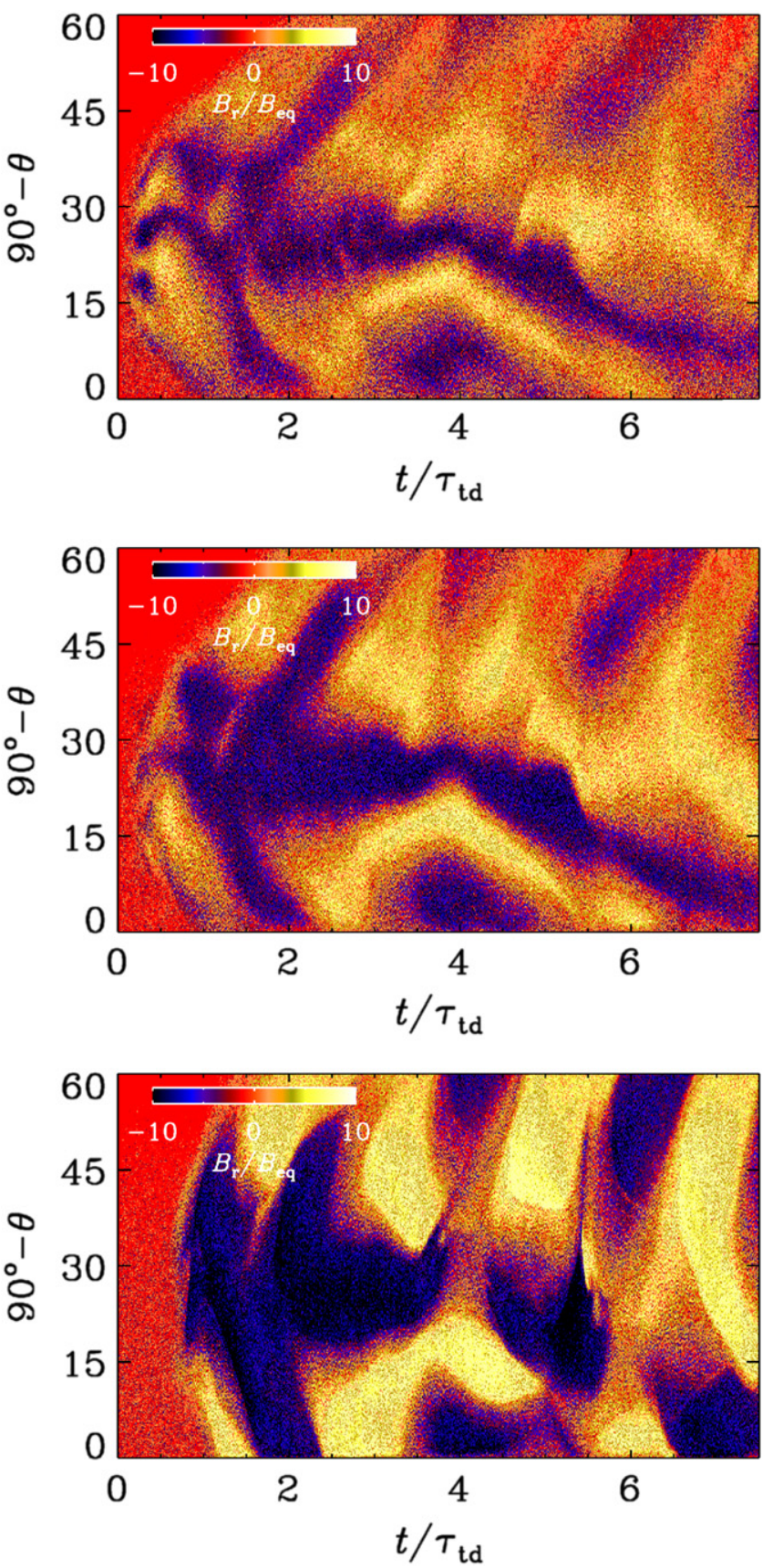

Figure 1. Butterfly diagram for our reference run (Run D2 with $\left.\Gamma_{\rho}=450\right)$ at $r / R=0.75$ (top), 0.8 (middle), and 0.95 (bottom).

active layer, which is now rather thin. This can lead to a larger number of toroidal flux belts (Moss et al. 1990). Another reason could be the comparatively short run time $\left(6.5 \tau_{\mathrm{td}}\right)$, which might imply that the field is still in a transient. However, in view of the comparatively large spatial resolution $(256 \times 1152 \times 1152$ mesh points), longer runs become computationally prohibitive. Note also the occurrence of sharp structures in the bottom panel of Figure 1 at $t / \tau_{\mathrm{td}} \approx 5.5$ (and also $\approx 3.5$ ). This rapid time variation is a consequence of plotting the field at fixed values of $\phi$ (here $\phi=0$ ) and the fact that the non-axisymmetric structures drift in $\phi$ (here in the westward direction).

The observed dynamo wave is generated by an $\alpha^{2}$ dynamo. The fact that $\alpha^{2}$ dynamos with nonuniform $\alpha$ distribution can 

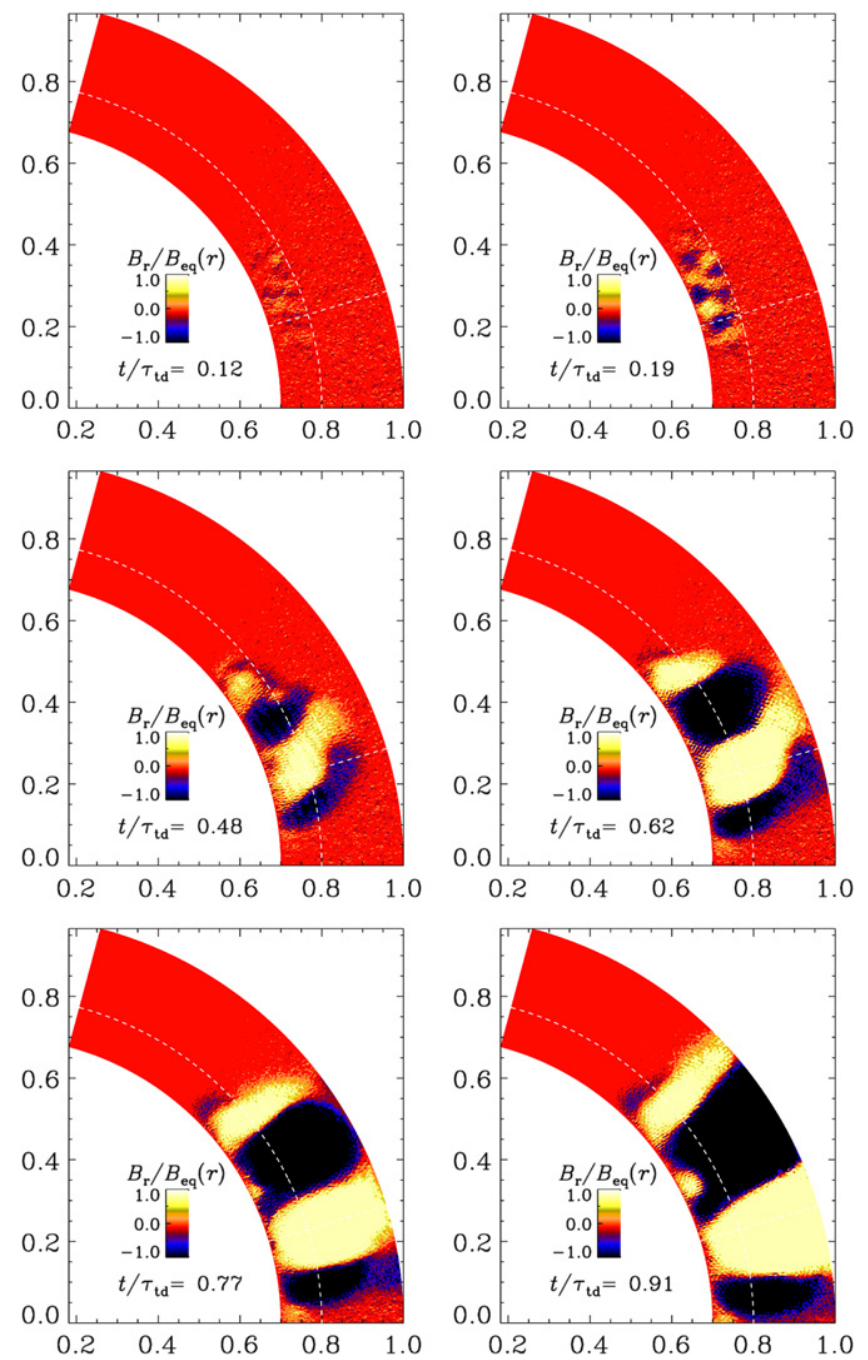

Figure 2. Meridional cross-sections of $\bar{B}_{r} / B_{\text {eq }}$ at different times for Run Q2.

be oscillatory was known for some time (Shukurov et al. 1985; Baryshnikova \& Shukurov 1987; Stefani \& Gerbeth 2005), but their migratory properties were first pointed out by Mitra et al. (2010). Not surprisingly, the magnetic field is generated in the lower layer, as can be seen from Figure 2 where we show meridional cross-sections of $B_{r}(r, \theta) / B_{\text {eq }}(r)$ at $\phi=0$ at six different times. In this sequence we have chosen a fixed color scale that saturates at the equipartition level. This allows us to see at which times and heights the field reaches equipartition. However, to see the spatial variation of the field, we show in Figure 3 the first and last times with a color range that saturates at 0.1 and 10 times the equipartition value.

We note that during the early growth phase of the dynamo, the magnetic field grows exponentially and the non-dimensional growth rate is of the order of 0.1 (in units of the inverse turbulent diffusion time). It increases with magnetic Reynolds number (cf. Runs R1 and R2), which might be related to the possibility of small-scale dynamo action. This is supported by the fact that the growth rate is not increasing with helicity (cf. Runs D2 and D4).

\subsection{Spot Formation}

Next, we consider the surface appearance of the radial magnetic field. We see the formation of structures at low
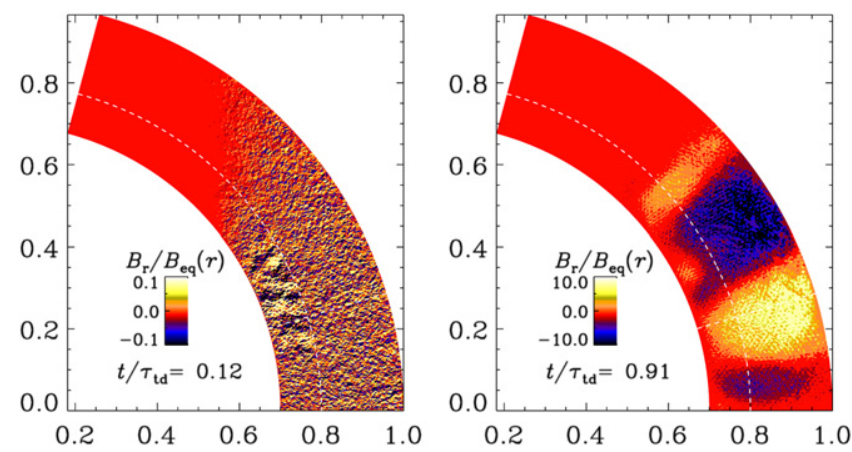

Figure 3. Similar to the first and last panels of Figure 2, but the range of the color scale is adapted to the actual extrema.

latitudes in less than a turbulent diffusive time. At first a few bipolar regions form. As time goes on, these structures move, rotate, and expand, and after a long enough time they form a strong field concentration which move toward the equator and forms three band-like structures with opposite polarities (see Figures 4 and 5). A similar behavior was also observed by Mitra et al. (2014); see Figures 3 and 4 of their paper.

Figures 4 and 5 illustrate the time evolution of our reference simulations (Runs Q2 and D2) with $\Gamma_{\rho}=450$. As one can see, at early times of bipolar spot formation, the two polarities are very close to each other. One sees that each polarity consists of a core with strong field and a shadow around it with weaker field. As time elapses, both core and the shadow expand but the speed of the expansion of the shadow is larger than the speed of expansion of the core. This implies that two polarities start moving apart form each other. The rest of the evolution is somewhat different for Runs Q2 and D2. For Run Q2, the bipolar spot orientation is preferentially in the azimuthal direction, both at early and later times. For Run D2, on the other hand, the spots tilt in such a way that the part of the structure with the same polarity tends to occupy a certain latitudinal band, while that of the opposite orientation occupies a band at a different latitude; see Figure 5. This global structure remains without change, no matter how much time passes.

The time dependence of magnetic energy and its radial profile are shown in Figure 6 for different normalizations. Different colors correspond to different degrees of stratification. Earlier studies have demonstrated the importance of stratification in the formation of structures through NEMPI. In fact, by increasing the stratification, the structures were found to be more intense. For the highest stratification $\left(\Gamma_{\rho}=1400\right)$, the total magnetic energy becomes somewhat larger than for $\Gamma_{\rho}=450$, although the strength of the spots is about the same. This suggests that there might be an upper limit for the density contrast. A similar effect was also observed in the work of Jabbari et al. (2014), which they referred to as gravitational quenching, which saturates or even suppresses NEMPI. We must also remember that NEMPI can only work in regions where the magnetic field relative to the equipartition value is in the optimal range (Kemel et al. 2012; Brandenburg et al. 2014; Losada et al. 2014). However, in our strongly stratified system, the regions where this would be the case can become rather shallow. This gives another geometric constraint on the possibility of NEMPI, which was already discussed by Losada et al. (2014) in connection with polytropic stratifications, where this limitation can become particularly severe. Further 

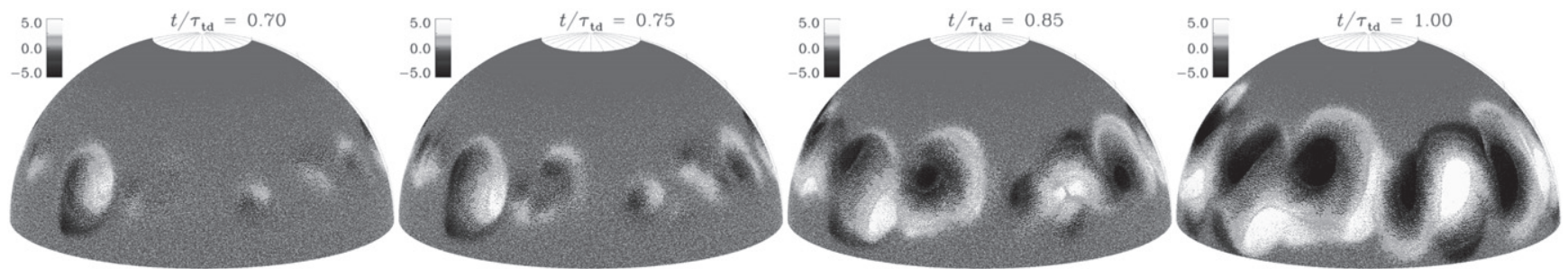

Figure 4. Time evolution of $B_{r} / B_{\mathrm{eq}}$ at $r / R=0.98$ for a simulation with $\Gamma_{\rho}=450$ for Run Q2.
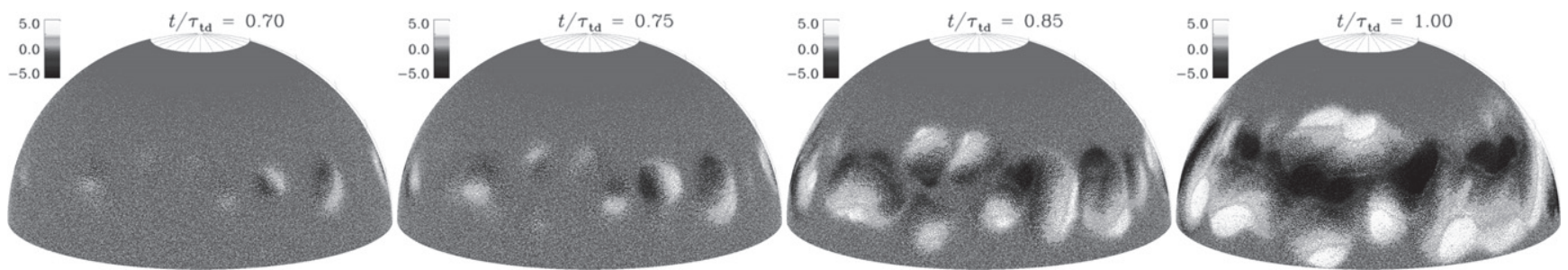

Figure 5. Same as Figure 4, but for Run D2.
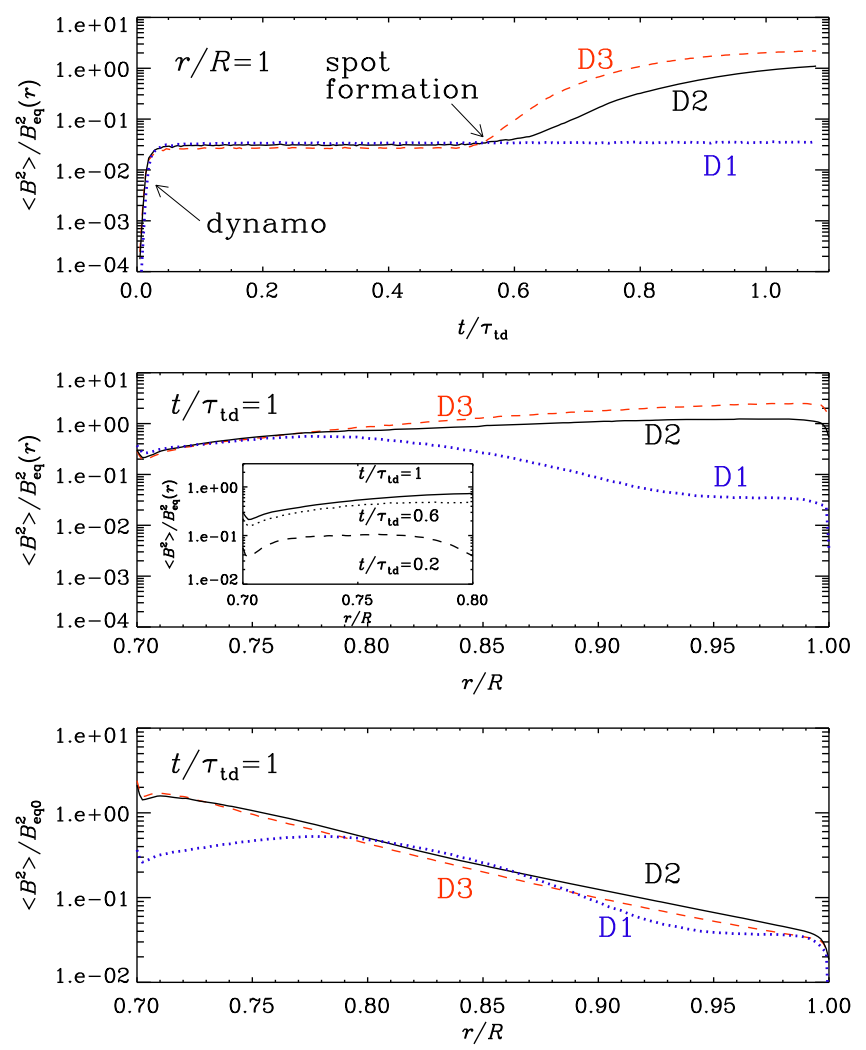

Figure 6. Upper panel: time evolution of $\left\langle\boldsymbol{B}^{2}\right\rangle / B_{\mathrm{eq}}^{2}(r)$ for Runs D1 (dotted, blue), D2 (solid, black), and D3 (dashed, red). Middle panel: radial dependence of $\left\langle\boldsymbol{B}^{2}\right\rangle / B_{\mathrm{eq}}^{2}(r)$ at $t / \tau_{\mathrm{td}}=1$ for Runs D1-D3. The inset shows the radial dependence of $\left\langle\boldsymbol{B}^{2}\right\rangle / B_{\text {eq }}^{2}(r)$ for Run D2 inside the $r / R<0.8$ (helical zone) at $t / \tau_{\mathrm{td}}=0.2$ (dotted), 0.6 (dashed), and 1 (solid). Lower panel: radial dependence of $\left\langle\boldsymbol{B}^{2}\right\rangle / B_{\mathrm{eq} 0}^{2}$ for fixed normalization.

studies are needed to understand the implications of this geometric effect.

The middle panel of Figure 6 shows that in the deeper parts $(r / R \leqslant 0.77)$, the normalized magnetic field strength is virtually independent of stratification. This demonstrates that the dynamo is not affected by stratification or the resulting spot formation. In the upper layers, on the other hand, the ratio
$\left\langle\boldsymbol{B}^{2}\right\rangle / B_{\mathrm{eq}}^{2}(r)$ varies significantly between the runs with different stratification. This is mainly a consequence of the strong variation of $B_{\mathrm{eq}}$, which can be seen from the bottom panel where $\left\langle\boldsymbol{B}^{2}\right\rangle / B_{\text {eq0 }}^{2}$ is shown.

Figure 7 shows the time evolution of $B_{r} / B_{\text {eq } 0}$ in the $r \phi$ plane along the active region belt $\left(\theta=22^{\circ}\right)$. One can see the formation of dynamo-generated large-scale field at the bottom and an intense magnetic field concentration at the surface layer. There is a similarity between Figures 7 and 8 of Mitra et al. (2014), because in both cases there appears to be a mechanism that concentrates the dynamo-generated sub-equipartition field in the deeper parts into super-equipartition field in the upper.

\subsection{Inclination Angle}

Our spots show a systematic east-west orientation with negative vertical field on the left and positive values on the right. In addition, some of the regions also show a certain tilt, although the apparent yin-yang structure makes it hard to say whether the tilt angle is positive or negative. Most of the bipolar regions are oriented in a similar fashion, although there is also a large fraction of spots that show random orientation. Parker (1955) suggested that sunspot pairs are produced by the buoyant rise of a flux tube, which takes the form of an $\Omega$ loop near the surface. To see whether this is also the case in the present simulations, we show $B_{r} / B_{\text {eq }}$ at the surface together with field vectors projected onto the horizontal plane (Figure 8). Note that the vectors tend to point in the negative $\phi$ direction, i.e., the azimuthal field points to the left. In most of the bipolar spots in this figure, $B_{r} / B_{\text {eq }}$ tends to be positive on the righthand side of the spot (pointing upward) and negative on the left-hand side of the spot (pointing downward). This corresponds to the expected $\Omega$ loop scenario.

\subsection{Dependence on $r_{\star}, \sigma_{\max }$, Reynolds Number, Stratification, and Scale Separation}

We recall that we adopt a similar forcing setup as Mitra et al. (2014) with a transition at a radius $r_{\star}$ from helical forcing in the deeper parts to non-helical in the upper parts. Mitra et al. (2014) found that, when the border is moved closer to the bottom of the convection zone, the structures appear later. This 

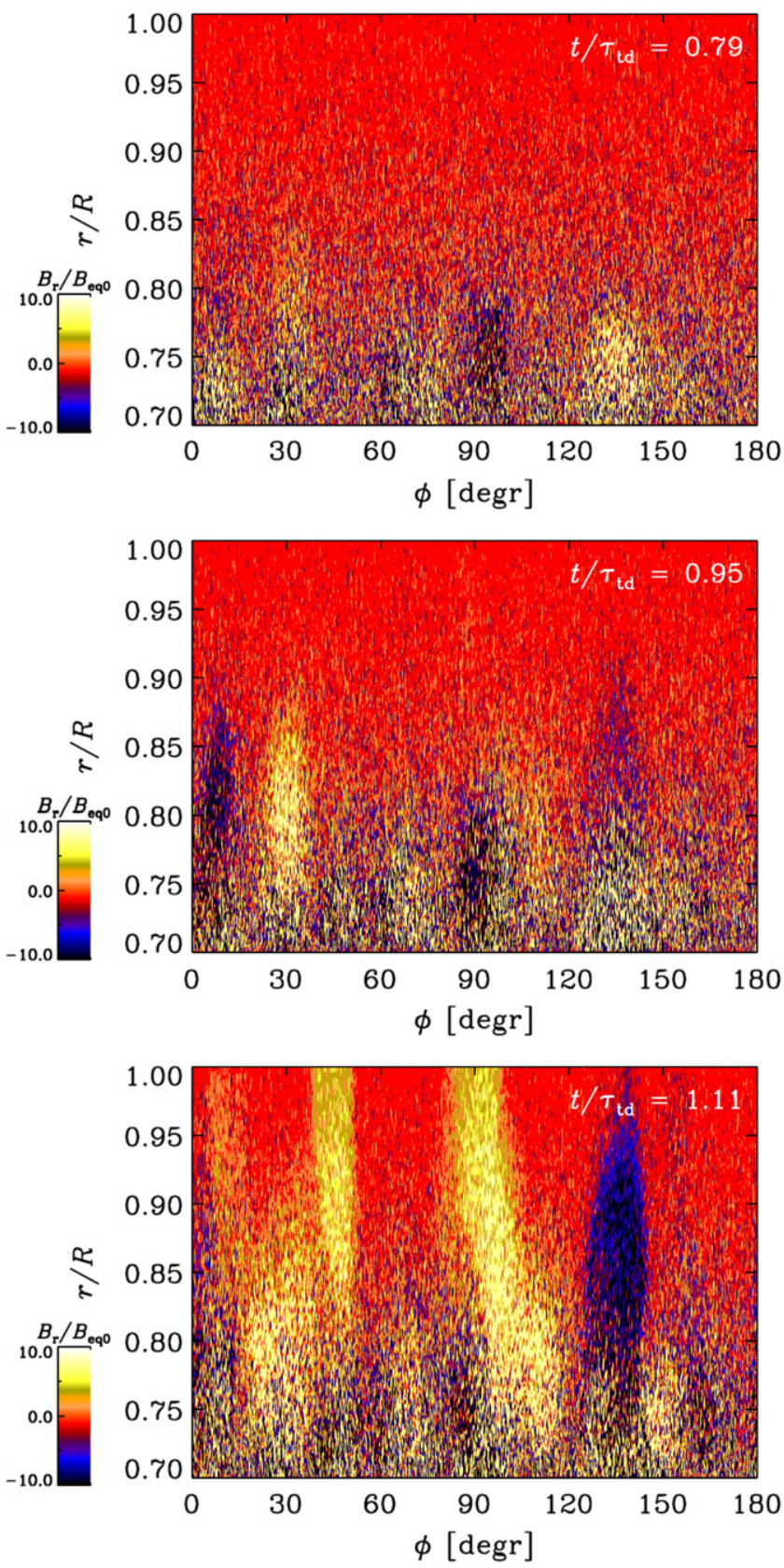

Figure 7. $r \phi$ projection of $B_{r} / B_{\text {eq0 }}$ along the active region belt for Run D2.

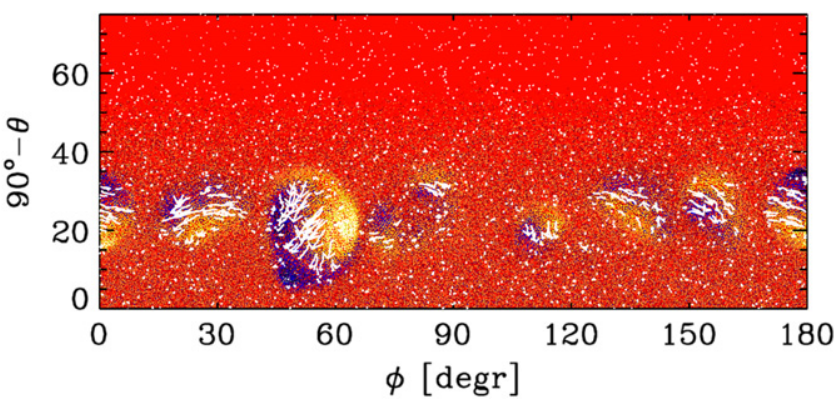

Figure 8. Mercator projection of $B_{r} / B_{\text {eq }}$ with $\left(B_{\theta}, B_{\phi}\right)$ vectors superimposed just below the surface at $t / \tau_{\mathrm{td}}=0.7$ for Run Q2. is due to the fact that it takes a longer time for dynamo to affect the upper layers. Similar behavior was observed when the helicity parameter $\sigma_{\max }$ is decreased from its maximum value of 1. In such a case the formation of structures occurs again with time delay, which is due to the weaker dynamo.This is shown in Figure 9, where we present the time evolution of $B_{r} / B_{\text {eq }}$ at $r / R=0.98$ for Run D5 with $\sigma_{\max }=0.5$. One can see the appearance of spots at later times $\left(t / \tau_{\mathrm{td}} \approx 2\right)$. Figure 9 also illustrates the fact that by weakening the helicity the spots become more intense and regular in comparison with the fully helical case (compare with Figure 4). However, the present simulations show that the dynamo growth rate does slightly increase with decreasing helicity; cf. Runs D2 and D4 or D5. On the other hand, lowering the value of $r_{\star}$ does lead to a small decrease of the growth rate; cf. Runs Q2 and Q4 as well as Runs $\mathrm{H} 2$ and $\mathrm{H} 4$.

The effect of stratification on the formation of the spot is shown in Figure 10. For the lowest density contrast, no structures form, independently of time, size of the shell, value of Reynolds number, and position of the border between helical and non-helical turbulence. This confirms that stratification plays a crucial role in the formation of bipolar regions. Simulations with different density contrasts $\left(\Gamma_{\rho}\right.$ between 2 and 1450) show that bipolar structures form for $\Gamma_{\rho}$ larger than 30. We note that for weak stratification $\left(\Gamma_{\rho}=30\right.$ for instance) spots appear only at late times $\left(t / \tau_{\mathrm{td}} \approx 3\right)$, while for stronger stratifications $\left(\Gamma_{\rho}=70\right)$ they appear earlier $\left(t / \tau_{\mathrm{td}} \approx 1\right)$ and are more concentrated. In the deeper parts $(r / R=0.75)$, on the other hand, the magnetic field evolution for Run Q1 with weak stratification is similar to that of Run Q2, which demonstrates that the dynamo is not effected by the strength of stratification. One also sees that the late time evolution of the magnetic field is similar for Runs Q1 and Q2, even though spots form in Run Q2, but not in Q1. This suggests that the formation of spots does not affect the dynamo. In spherical geometry, it is possible to investigate the effect of different $\phi$ extent on the formation of structures. Not surprisingly, it turns out that for small $\phi$ extent (below $\pi / 6$ ) the size of the spots is limited by the domain size because the formation of strongly innomogenous structures requires strong-scale separation between the energy-carrying eddies and the domain.

\subsection{Effective Magnetic Pressure}

To assess whether NEMPI is operating, we now calculate the effective magnetic pressure, following Brandenburg et al. (2012) and adapting the formulation to the spherical case. The total stress from the fluctuating velocity and magnetic fields is given by

$$
\bar{\Pi}_{i j}^{f}=\bar{\rho} \overline{u_{i} u_{j}}+\frac{1}{2} \delta_{i j} \overline{\boldsymbol{b}^{2}}-\overline{b_{i} b_{j}},
$$

where the superscript $f$ denotes the fluctuating terms. In the following we only need the three diagonal components of $\bar{\Pi}_{i j}^{f}$, which we denote by $\bar{\Pi}_{i}^{f}$, where $i$ refers to $r, \theta$, or $\phi$. As we are interested in the contribution from the part that results from the mean field, we should calculate the stress also for zero mean 

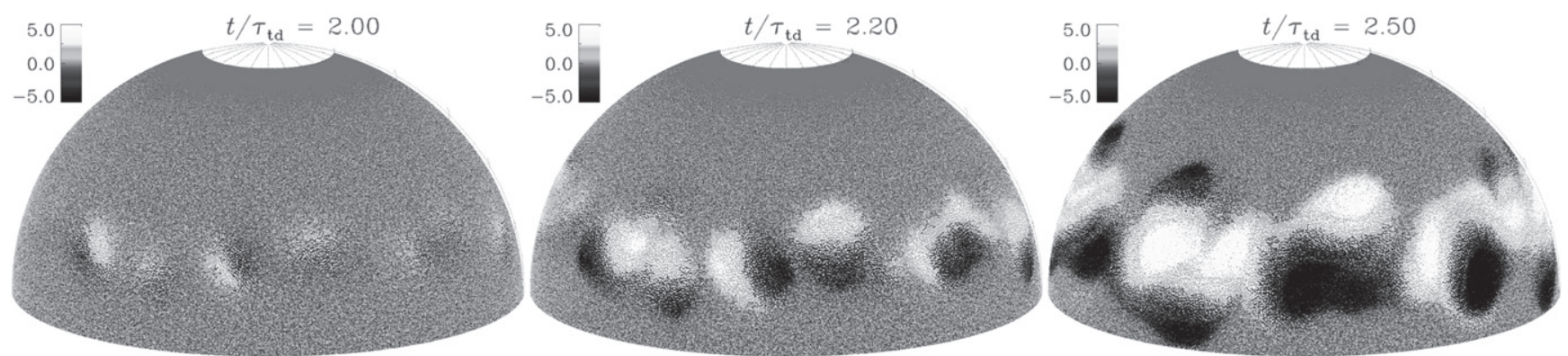

Figure 9. Time evolution of $B_{r} / B_{\mathrm{eq}}$ at $r / R=0.98$ for a simulation with $\sigma_{\max }=0.5$ (Run D5).
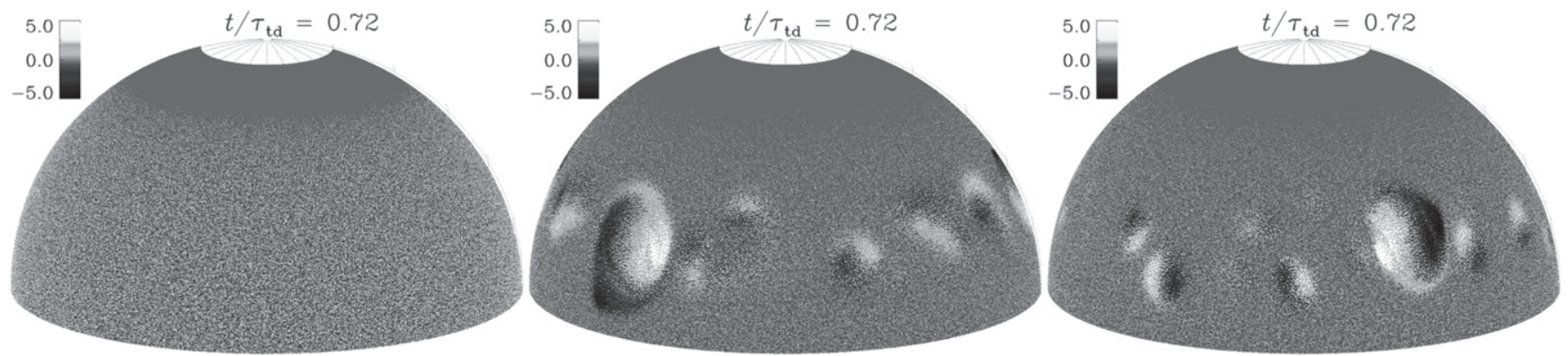

Figure 10. $B_{r} / B_{\text {eq }}$ at $r / R=0.98$ for simulations with different stratifications for Runs Q1, Q2 and Q3 with density contrasts 2, 450 and 1400 from left to the right, respectively.

field and subtract it from the total stress, so

$$
\Delta \bar{\Pi}_{i}^{f}=\bar{\rho}\left(\overline{u_{i}^{2}}-\overline{u_{0 i}^{2}}\right)+\frac{1}{2}\left(\overline{\boldsymbol{b}^{2}}-\overline{\boldsymbol{b}_{0}^{2}}\right)-\left(\overline{b_{i}^{2}}-\overline{b_{0 i}^{2}}\right),
$$

where subscript 0 refers to the case with zero mean magnetic field. But as the background field is here dynamo-generated, we use the values of the related quantities in the upper part of the domain (non-helical part) at early times. This gives us the possibility to estimate the effective magnetic pressure in spherical geometry with dynamo-generated magnetic field. In Equation (14), $\Delta \bar{\Pi}_{i}^{f}$ denotes the three diagonal components of the tensor $\Delta \bar{\Pi}_{i j}^{f}$. In the mean-field description, it depends on the mean magnetic field $\overline{\boldsymbol{B}}$ and is parameterized as

$$
\Delta \bar{\Pi}_{i j}^{f}=\left(q_{s} \hat{\beta}_{i} \hat{\beta}_{j}-\frac{1}{2} q_{p} \delta_{i j}+q_{g} \hat{g}_{i} \hat{g}_{j}\right) \overline{\boldsymbol{B}}^{2},
$$

where $\hat{\beta}_{i}$ and $\hat{g}_{i}$ are the unit vectors along $\overline{\boldsymbol{B}}$ and $\boldsymbol{g}$, respectively. The effective magnetic pressure is defined as a sum of nonturbulent and turbulent contributions:

$$
\mathcal{P}_{\text {eff }}=\frac{1}{2}\left(1-q_{p}\right) \beta^{2},
$$

where $\beta^{2}=\bar{B}^{2} / B_{\text {eq }}^{2}$ and $q_{p}$ is a turbulent transport coefficent that depends on the mean magnetic field and can be computed from the DNS as

$$
q_{p}=-\frac{1}{\overline{\boldsymbol{B}}^{2}}\left[\Delta \bar{\Pi}_{\theta}^{f}+\Delta \bar{\Pi}_{\phi}^{f}-q_{s}\left(\bar{B}_{\theta}^{2}+\bar{B}_{\phi}^{2}\right)\right],
$$

with

$$
q_{s}=\left(\Delta \bar{\Pi}_{\theta}^{f}-\Delta \bar{\Pi}_{\phi}^{f}\right) /\left(\bar{B}_{\theta}^{2}-\bar{B}_{\phi}^{2}\right),
$$

and

$$
q_{g}=\frac{1}{\overline{\boldsymbol{B}}^{2}}\left[-\Delta \bar{\Pi}_{r}^{f}+q_{s} \bar{B}_{r}^{2}-\frac{1}{2} q_{p} \overline{\boldsymbol{B}}^{2}\right] .
$$

Previous studies (Brandenburg et al. 2012; Käpylä et al. 2012a) in Cartesian geometry have shown that $q_{s}$ and $q_{g}$ are very close to zero. This is also confirmed by the present simulations, where $\Delta \bar{\Pi}_{\theta}^{f}-\Delta \bar{\Pi}_{\phi}^{f}$ is found to correlate poorly with $\bar{B}_{\theta}^{2}-\bar{B}_{\phi}^{2}$. We therefore ignore $q_{s}$ and $q_{g}$ in most of the following.

Next, we subtract the time average of an early time interval between times $t_{1}$ and $t_{2}$ and compute the diagonal components of the change of the stress as

$$
\begin{aligned}
\Delta \bar{\Pi}_{i}^{f}(r, \theta, t)= & \bar{\Pi}_{i}^{f}(r, \theta, t)-\frac{1}{t_{2}-t_{1}} \\
& \times \int_{t_{1}}^{t_{2}} \bar{\Pi}_{i}^{f}\left(r, \theta, t^{\prime}\right) d t^{\prime},
\end{aligned}
$$

where $t_{1}=0.1 \tau_{\mathrm{td}}$ and $t_{2}=0.5 \tau_{\mathrm{td}}$ denote the time interval over which the turbulence in the upper layer is not yet affected by the mean magnetic field. We thus compute

$$
q_{p}=-\left(\Delta \bar{\Pi}_{\theta}^{f}+\Delta \bar{\Pi}_{\phi}^{f}\right) / \bar{B}^{2},
$$

so we get $\mathcal{P}_{\text {eff }}=\frac{1}{2}\left(1-q_{p}\right) \beta^{2}$.

In Figure 11 we show $\mathcal{P}_{\text {eff }}$ versus $t$ and $\theta$ for $r / R=0.85$ using Runs Q1, Q2, and Q3. The bottom panels show scatter plots of $\mathcal{P}_{\text {eff }}(\beta)$. It is customary to fit such data to an expression of the form (Kemel et al. 2012)

$$
q_{p}(\beta)=\beta_{*}^{2} /\left(\beta_{p}^{2}+\beta^{2}\right),
$$



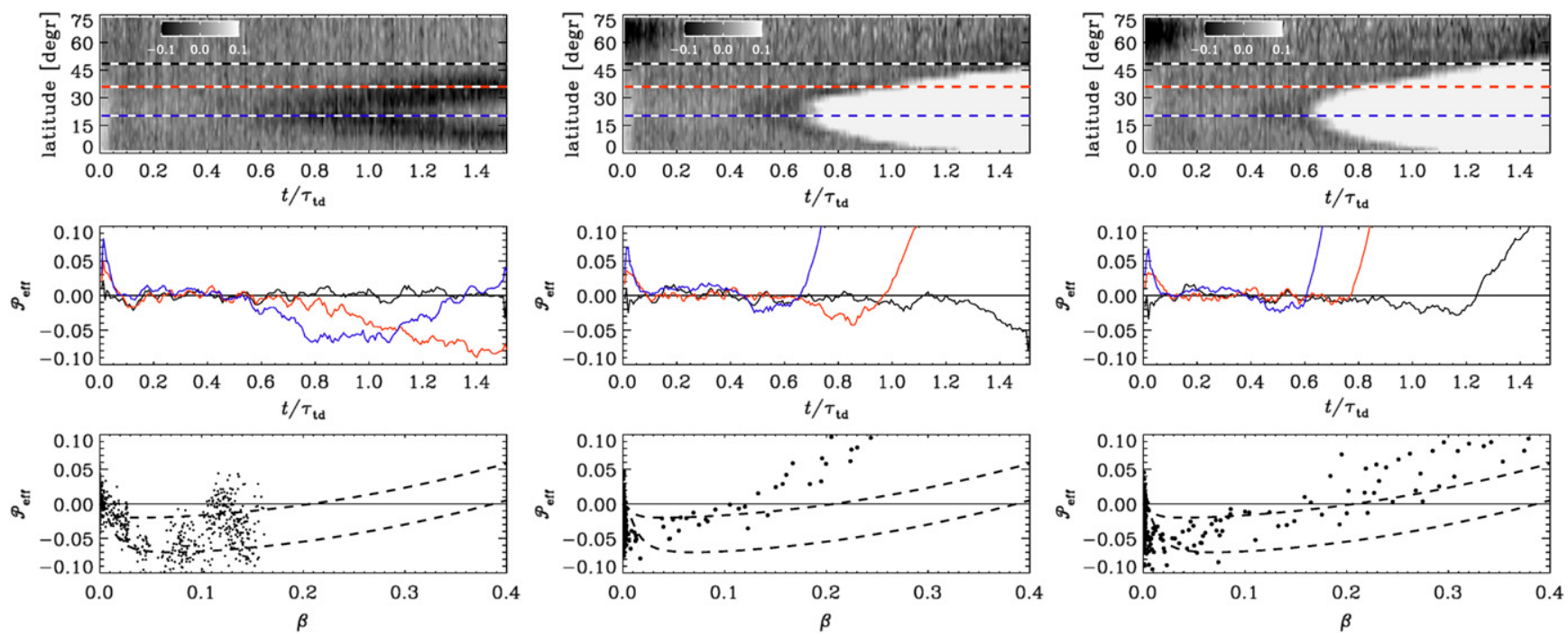

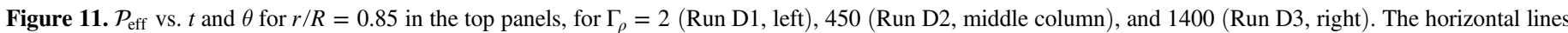

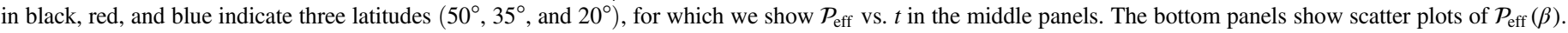
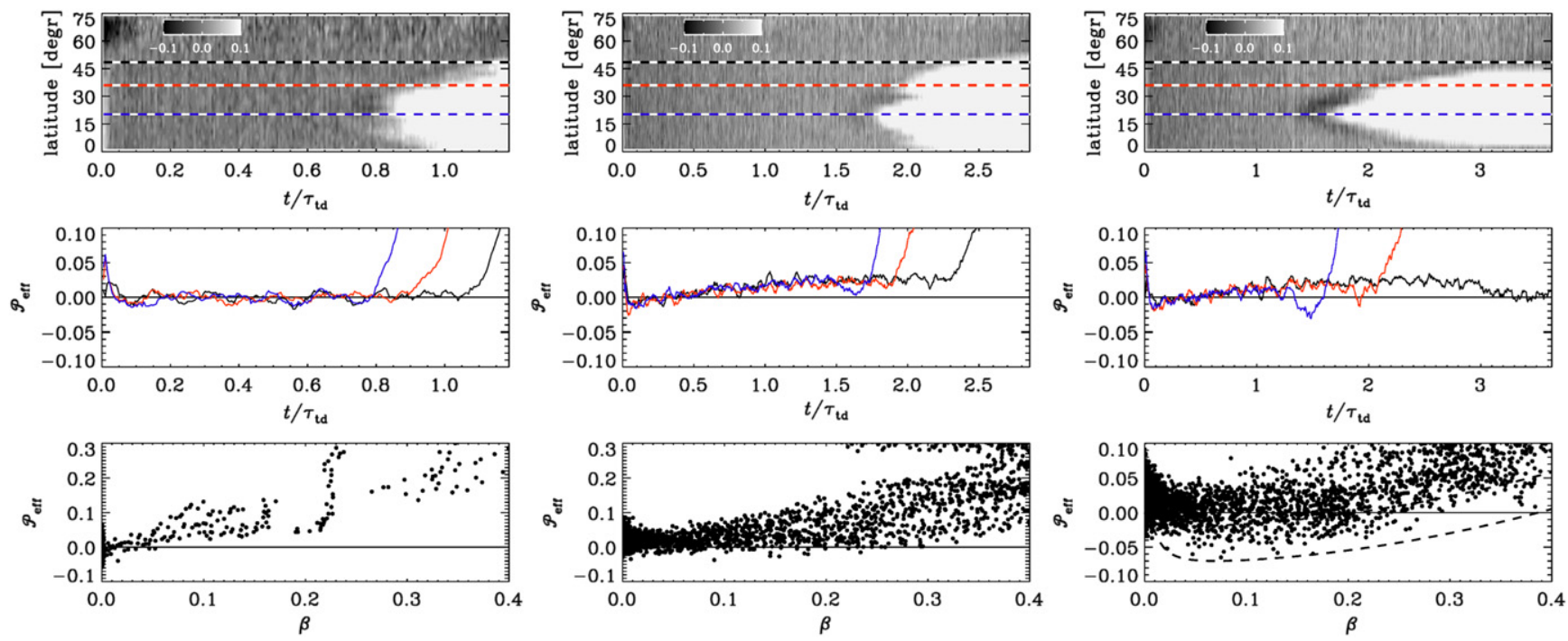

Figure 12. Similar to Figure 11, but for $r / R=0.98$ and for $\Gamma_{\rho}=450$ at $\sigma_{\max }=1$ (left), $\sigma_{\max }=0.5$ (middle), and $r / R=0.85$, again with $\sigma_{\max }=0.5$ (right).

where $\left(\beta_{*}, \beta_{p}\right)$ is a set of fit parameters. They have previously been determined for Cartesian simulations with an imposed magnetic field (Brandenburg et al. 2012). In Figure 11, the dashed lines show for comparison the result for two representations of Equation (22) with $\left(\beta_{*}, \beta_{p}\right)=(0.39,0.013)$ and $(0.21,0.008)$ for curves with the deeper and less deep minimum, respectively. Neither of the curves fit the data points well. Nevertheless, it is important to note that $\mathcal{P}_{\text {eff }}$ is always negative for $\beta<0.1$, although in the runs with stronger stratification the number of such points is rather small.

One may speculate that in Runs D2 and D3, which produce strong fields, there are two stages of magnetic field concentrations. At early times, the field is below the equipartition field strength, so NEMPI works and the effective magnetic pressure is negative. At later times, when the field is of the order of or larger than the equipartition field strength, the effective magnetic pressure becomes positive and the standard magnetic buoyancy instability might play a role in bringing magnetic field to the surface, perhaps as what is seen in Figure 7. We may conclude that, while there is evidence for negative values of $\mathcal{P}_{\text {eff }}$, there may still be other effects playing important roles. In particular the formation of relatively sharp boundaries of our spots, which is a marked feature of both the present calculations and those of Mitra et al. (2014), may be due to such a new effect. It is reminiscent of the appearance of sharp structures as a result of ambipolar diffusion under laminar conditions (Brandenburg \& Zweibel 1994). If so, it may be related to mean-field terms in the induction equation rather than the momentum equation.

In the near-surface layers, on the other hand, there is no evidence for negative effective magnetic pressure, as can be seen from Figure 12 (left panels), where we show the results for $\mathcal{P}_{\text {eff }}$ at $r / R=0.98$ and for $\Gamma_{\rho}=450$. This is however consistent with the idea that spots are formed mainly due to suction from deeper layers (Brandenburg et al. 2014). For Run D5 with $\sigma_{\max }=0.5$, the results are similar to Run D2, although $\mathcal{P}_{\text {eff }}$ is slightly smaller (middle panels of Figure 12) 

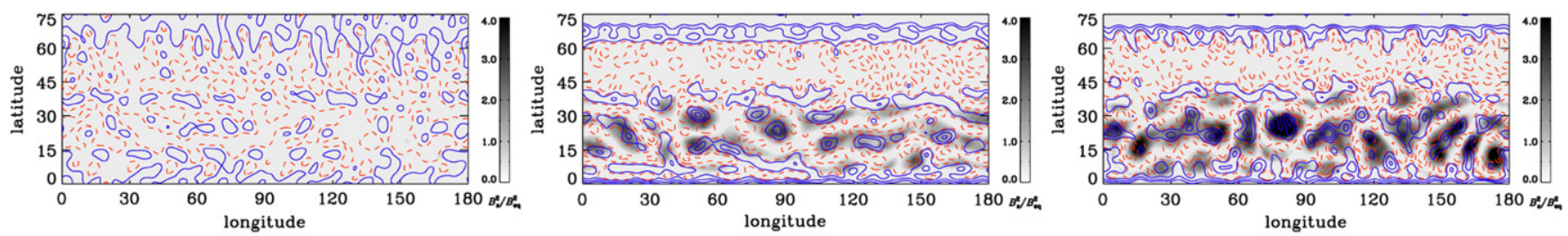

Figure 13. Contours of negative (blue, solid lines) and positive (red, dashed) vertical velocity $\left\langle U_{r}\right\rangle_{k R<50}$ superimposed on a gray-scale representation of $\left\langle B_{r}\right\rangle_{k R<100}^{2} / B_{\text {eq }}^{2}(r)$ in Mercator projection at $r / R=0.85$ and $t / \tau_{\mathrm{td}}=0.7$ for Run D1 (left panel, Run D2 (middle panel) and Run D3 (right panel)).

and at $r / R=0.85$ the values of $\mathcal{P}_{\text {eff }}$ are generally less negative (right panels of Figure 12).

To check whether there are downflows at greater depth where NEMPI can act, we compare in Figure 13 for Runs D1, D2, and, D3 contours of negative and positive vertical velocity, $\left\langle U_{r}\right\rangle_{k R<50}$, superimposed on a gray-scale representation of $\left\langle B_{r}\right\rangle_{k R<100}^{2} / B_{\mathrm{eq}}^{2}(r)$ at $r / R=0.85$. Here, $\langle\cdot\rangle_{k R}$ denotes Fourier filtering, applied to obtain smoother contours. (For $B_{r}$ we also apply some filtering, but only above $k R=100$ to eliminate patterns on the scale of the forcing.) We see that for sufficiently strong stratification (Runs D2 and D3), there are indeed many locations where the field is strong and $\left\langle U_{r}\right\rangle_{k R<50}$ is negative, but the correlation is not very strong. However, the speed of the downflows increases with increasing stratification (compare the last two panels of Figure 13). Furthermore, since the field strength exceeds the equipartition value even at this greater depth, NEMPI must have seized to work. Nevertheless, it could have operated at earlier times when the field was weaker.

Finally, a comment regarding the possible importance of the $q_{s}$ term is in order. As we have mentioned above, $q_{s}$ cannot be determined owing to the poor correlation between $\Delta \bar{\Pi}_{\theta}-\Delta \bar{\Pi}_{\phi}$ and $\bar{B}_{\theta}^{2}-\bar{B}_{\phi}^{2}$. However, at $r / R=0.85$ and near $35^{\circ}$ latitude, the correlation is not quite as poor and $q_{s}$ can be determined in the time interval $0.7<t / \tau_{\mathrm{td}}<0.85$, where for Run D2 $q_{s} \approx 20$ is found. On the other hand, in the neighborhood of this latitude, $q_{s}$ turns out to be in the range $0<q_{s}<20$. The corresponding values of $q_{g}$ are found to be in the range from -5 to 10. Furthermore, if the $q_{s}$ term in Equation (19) were ignored, we find values in the range $-5<q_{g}<0$. Interestingly, MFS of NEMPI have shown earlier that values in the range $-10<q_{g}<10$ do not significantly affect the growth rate (Käpylä et al. 2012a), suggesting that $q_{g}$ remains subdominant, regardless of whether or not the $q_{s}$ term is taken into account.

\subsection{High-latitude Spots}

Before concluding, let us return once more to the occurrence of magnetic spots and its dependence on the parameter $n$ in Equation (12). When the helicity is large at high latitudes, i.e., when we choose the helicity profile with $n=0$, magnetic spots are found to form close to the poles in a fashion reminiscent of recent simulations by Yadav et al. (2015). Figure 14 presents the formation of bipolar spots near the pole for run $\mathrm{H} 2$. By contrast, the work of Yadav et al. (2015) showed just a single spot. However, in both cases the underlying dynamo process is a distributed one, so the lower boundary at the bottom of the domain is not critical for its operation.

The results of the simulations with $n=0$ show similar behavior and parameter dependence as the case with $n=6$. As for $n=6$, for weaker stratification no spots form and for

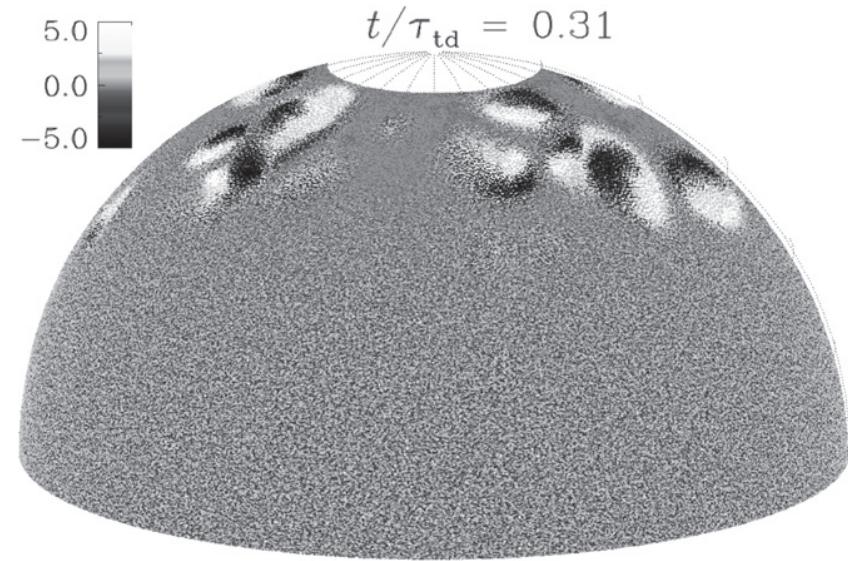

Figure 14. Formation of the high-latitude spots for the case $n=0$ (Run H2).

smaller $\sigma_{\max }$ and deeper $r_{\star}$, the structures form with a time delay.

\section{CONCLUSIONS}

The present work has demonstrated that in a strongly stratified two-layer spherical model with helical turbulence in the lower layer and non-helical turbulence in the upper one, the $\alpha^{2}$ dynamo produces large-scale magnetic fields that develop sharp spot-like structures at the surface. This extends the results of Mitra et al. (2014) to spherical geometries. We therefore see for the first time that the bipolar magnetic spots have a finite size that is not limited by the domain size as in the work of Mitra et al. (2014). However, contrary to earlier expectations (Kemel et al. 2012; Brandenburg et al. 2014), the size of these structures exceeds the local value of the density scale height by much more than the earlier expected value of about ten.

In our present simulations the dynamo (caused by the $\alpha^{2}$ dynamo) was very efficient, because the forcing was assumed to be fully helical. In reality, the helicity is caused by the combined action of rotation and stratification (Krause \& Rädler 1980). In this sense, dynamos do depend on stratification, contrary to the present case with helical forcing, where this was found to be not the case; see Section 3.2. As a consequence of the strong helicity, the resulting large-scale field is rather strong and the magnetic spots begin to fill eventually the entire horizontal surface. This is also what is expected for very active stars where the filling factor of the surface magnetic field is known to reach unity as the star becomes more active (Saar \& Linsky 1985). Conversely, to model sunspots, which are much smaller, we expect that we would need to decrease the fractional helicity below the values explored in the present work.

Visualizations of the three-dimensional magnetic field structure in convectively driven dynamos has revealed the 
formation and subsequent rise of serpentine-shaped flux tubes (Fan \& Fang 2014; Nelson \& Miesch 2014). Our work has now shown that strong stratification may provide the key to understanding how such structures can experience re-amplification, which is required if they are to be responsible for spot formation.

While it is clear that strong stratification and large scale separation between the turbulent integral scale and the size of the box are essential elements behind spot formation, the results presented here are different from our earlier findings that were obtained under more idealized conditions such as the use of an imposed magnetic field. However, like the earlier results of Mitra et al. (2014) we find again evidence for downflows below the sites of spot formation and, in particular, the formation of sharply bounded structures. The latter is reminiscent of the appearance of sharp structures as a result of ambipolar diffusion under laminar conditions (Brandenburg $\&$ Zweibel 1994). This raises the question, whether for strongly stratified turbulence the effective magnetic diffusivity can attain a nonlinear dependence on the magnetic field that is similar to that of ambipolar diffusion, i.e., it increases with increasing field strength. To address these questions, it would be best to return to Cartesian geometry where it would be possible to determine turbulent transport coefficients with dedicated methods such as the test-field approach. This is however beyond the scope of the present work.

This work was supported in part by the Swedish Research Council Grants No. 621-2011-5076 and 2012-5797, the Research Council of Norway under the FRINATEK grant 231444, the Academy of Finland under the ABBA grant No. 280700, as well as the Russian Federal Program under the grant No. 14.578.21.0033. We acknowledge the allocation of computing resources provided by the Swedish National Allocations Committee at the Center for Parallel Computers at the Royal Institute of Technology in Stockholm and the National Supercomputer Centers in Linköping, the High Performance Computing Center North in Umeå, and the Nordic High Performance Computing Center in Reykjavik.

\section{REFERENCES}

Baryshnikova, I., \& Shukurov, A. 1987, AN, 308, 89

Brandenburg, A., Candelaresi, S., \& Chatterjee, P. 2009, MNRAS, 398, 1414
Brandenburg, A., Gressel, O., Jabbari, S., Kleeorin, N., \& Rogachevskii, I. 2014, A\&A, 562, A53

Brandenburg, A., Kemel, K., Kleeorin, N., Mitra, D., \& Rogachevskii, I. 2011, ApJL, 740, L50

Brandenburg, A., Kemel, K., Kleeorin, N., \& Rogachevskii, I. 2012, ApJ, 749, 179

Brandenburg, A., Kleeorin, N., \& Rogachevskii, I. 2010, AN, 331, 5

Brandenburg, A., Kleeorin, N., \& Rogachevskii, I. 2013, ApJL, 776, L23

Brandenburg, A., \& Zweibel, E. G. 1994, ApJL, 427, L91

Caligari, P., Moreno-Insertis, F., \& Schüssler, M. 1995, ApJ, 441, 886

Fan, Y., \& Fang, F. 2014, ApJ, 789, 11

Jabbari, S., Brandenburg, A., Kleeorin, N., Mitra, D., \& Rogachevskii, I. 2013, A\&A, 556, A106

Jabbari, S., Brandenburg, A., Losada, I. R., Kleeorin, N., \& Rogachevskii, I. 2014, A\&A, 568, A112

Käpylä, P. J., Brandenburg, A., Kleeorin, N., Mantere, M. J., \& Rogachevskii, I. 2012a, MNRAS, 422, 2465

Käpylä, P. J., Mantere, M. J., \& Brandenburg, A. 2012b, ApJL, 755, L22

Kemel, K., Brandenburg, A., Kleeorin, N., \& Rogachevskii, I. 2012, AN, 333, 95

Kleeorin, N., Mond, M., \& Rogachevskii, I. 1996, A\&A, 307, 293

Kleeorin, N., \& Rogachevskii, I. 1994, PhRvE, 50, 2716

Kleeorin, N. I., Rogachevskii, I. V., \& Ruzmaikin, A. A. 1989, SvAL, 15, 274

Kleeorin, N. I., Rogachevskii, I. V., \& Ruzmaikin, A. A. 1990, Sov. Phys. JETP, 70, 878

Kochukhov, O., Mantere, M. J., Hackman, T., \& Ilyin, I. 2013, A\&A, 550, A84

Krause, F., \& Rädler, K.-H. 1980, Mean-field Magnetohydrodynamics and Dynamo Theory (Oxford: Pergamon Press)

Losada, I. R., Brandenburg, A., Kleeorin, N., \& Rogachevskii, I. 2014, A\&A, 564, A2

Moss, D., Tuominen, I., \& Brandenburg, A. 1990, A\&A, 240, 142

Mitra, D., Brandenburg, A., Kleeorin, N., \& Rogachevskii, I. 2014, MNRAS, 445,761

Mitra, D., Tavakol, R., Käpylä, P. J., \& Brandenburg, A. 2010, ApJL, 719, L1

Nelson, N. J., \& Miesch, M. S. 2014, PPCF, 56, 064004

Parker, E. N. 1955, ApJ, 121, 491

Parker, E. N. 1979, ApJ, 232, 291

Rempel, M., \& Cheung, M. C. M. 2014, ApJ, 785, 90

Rogachevskii, I., \& Kleeorin, N. 2007, PhRvE, 76, 056307

Saar, S. H., \& Linsky, J. L. 1985, ApJL, 299, L47

Shukurov, A., Sokoloff, D., \& Ruzmaikin, A. 1985, MHD, 1, 9

Stefani, F., \& Gerbeth, G. 2005, PhRvL, 94, 184506

Stein, R. F., \& Nordlund, Å. 2012, ApJL, 753, L13

van Ballegooijen, A. A. 1984, in Proc. Conf. held in Sunspot, Small-Scale Dynamical Processes in Quiet Stellar Atmospheres, ed. L. K. Stephen (Aura), 260

Warnecke, J., Brandenburg, A., \& Mitra, D. 2011, A\&A, 534, A11

Warnecke, J., Losada, I. R., Brandenburg, A., Kleeorin, N., \& Rogachevskii, I. 2013, ApJL, 777, L37

Warnecke, J., Losada, I. R., Brandenburg, A., Kleeorin, N., \& Rogachevskii, I. 2015, arXiv: 1502.03799

Yadav, R. K., Gastine, T., Christensen, U. R., \& Reiners, A. 2015, A\&A, 573, A68 